\title{
A NOTE ON "REMARKS ON SOME INEQUALITIES FOR POSITIVE SEMIDEFINITE MATRICES AND QUESTIONS FOR BOURIN"
}

\section{JIANGUO ZHAO AND QI JIANG}

Abstract. Let $A_{i}, B_{i} \in \mathscr{M}_{n}$ be positive semidefinite matrices with $A_{i} B_{i}=B_{i} A_{i}(i=1,2, \cdots, m)$. Then

$$
\sigma\left(\left(\sum_{i=1}^{m}\left(A_{i} B_{i}\right)^{\frac{1}{2}}\right)^{r}\right) \prec_{w l o g} \sigma\left(\left(\sum_{i=1}^{m} A_{i}\right)^{\frac{r}{4}}\left(\sum_{i=1}^{m} B_{i}\right)^{\frac{r}{2}}\left(\sum_{i=1}^{m} A_{i}\right)^{\frac{r}{4}}\right),
$$

where $r \geqslant 1$. This result is a refinement of M. Hayajneh, S. Hayajneh and F. Kittaneh's result.

Mathematics subject classification (2010): 15A60, 15A18.

Keywords and phrases: Weak log-majorization, unitarily invariant norms, positive definite matrices.

\section{REFERENCES}

[1] K. M. R. AUdEnAERT, A norm inequality for pairs of commuting positive semidefinite matrices, Electron. J. Linear Algebra., 30, (2015), 80-84.

[2] R. Bhatia, Positive Definite Matrices, Princeton University Press, 2007.

[3] J. Bourin And M. UChIYAMA, A matrix subadditivity inequality for $f(A+B)$ and $f(A)+f(B)$, Linear Algebra Appl., 423, (2007), 512-518.

[4] M. Hayajneh, S. Hayajneh And F. Kittaneh, Remarks on some norm inequalities for positive semidefinite matrices and questions of Bourin, Math. Inequal. Appl., Preprint.

[5] F. HiAI, Log-majorizations and norm inequalities for exponential operators, In Linear Operators, Banach Center Publications 38, (1997), 119-181.

[6] D. HoA, An inequality for $t$-geometric means, Math. Inequal. Appl., 19(2), (2016), 765-768.

[7] M. Lin, Remarks on two recent results of Audenaert, Linear Algebra Appl., 489, (2016), 24-29.

[8] J. S. Matharu And J. S. Aujla, Some inequalities for unitarily invariant norms, Linear Algebra Appl., 436, (2012), 1623-1631.

[9] X. ZHAN, Matrix Theory, Higher Education Press, In Chinese, 2008. 